\title{
Internet-Based versus Center-Based Intervention Training for Parents of Young Children with Post-Digital Nanning Autism Syndrome (PDNAS): A Comparative Study
}

\author{
Hamidreza Pouretemad ${ }^{1}$, Saeid Sadeghi ${ }^{2,3}$, Bita Shalani ${ }^{4}$ \\ ${ }^{1}$ Institute for Cognitive and Brain Sciences, Shahid Beheshti University, Tehran, Iran. \\ ${ }^{2}$ Dept. of Clinical \& Health Psychology, Shahid Beheshti University, Tehran, Iran. \\ ${ }^{3}$ Center of Excellence in Cognitive Neuropsychology, Shahid Beheshti University, Tehran, Iran \\ ${ }^{4}$ Department of Psychology, Tarbiat Modares University, Tehran, Iran \\ Corresponding author: Professor Hamidreza Pouretemad; Email: h-pouretemad@sbu.ac.ir; Institute for Cognitive \\ and Brain Sciences, Shahid Beheshti University, Tehran, Iran
}

\section{Abstract \\ objectives}

Internet-based intervention approach is one novel strategy to train. However, only a few clinical trials have compared internet-based parent training intervention with an equal center-based intervention for children with ASD. The primary aim of this study was to compare treatment outcomes of an internet-based intervention with a center-based intervention for young children with Post Digital Nanning Autism Syndrome (PDNAS).

\section{Methods}

A total of 40 mother-young children with PDNAS dyads were assigned to the internet-based $(n=20)$ and to the center-based intervention $(n=20)$. Parents in both groups received intervention that involves 7 sessions (one session per week). Primary outcome measures were the Gilliam Autism Rating Scale - Second Edition (GARS-2), Repetitive Behavior Scale- Revised (RBS-R) and Parenting Stress Index (PSI).

\section{Results}

The analysis yielded no significant between-group difference for any of the pre- to postintervention measurements. At post-intervention both intervention conditions revealed significant symptoms changes compared to before the intervention. Also, the parental stress was significant related to the child's autism symptoms and repetitive behaviors severity.

Conclusion: Internet-based parent training intervention for young children with PDNAS and their parents is equally beneficial to regular center-based parent training intervention. These findings support the potential for using telehealth to provide research-based parent training interventions to any family that has access to the Internet.

Key words: Internet-Based Parent Training, Autism Symptoms, Repetitive Behaviors, PostDigital Nanning Autism Syndrome 


\section{Introduction}

Exposure of young children to digital devices (smartphones, tablets, televisions, etc.) has increased in recent years(Chang, Park, Yoo, won Lee, \& Shin, 2018; Kabali et al., 2015). Many of research has shown that early excessive screen time can cause symptoms of autistic behavior. Waldman et al. (2006) investigated whether does early childhood television viewing cause autism and they concluded that early childhood television viewing being an important trigger for autism (Waldman, Nicholson, \& Adilov, 2006). Heffler and Oestreicher (2016) proposed a model that seeks to explain how digital devices can cause autism spectrum disorder. In this model, excessive screen time in young children as an inappropriate environmental experience during critical periods of brain development and neuroplasticity leads to the specialization of brain non-social pathways and leads to the development of autism disorder (Heffler \& Oestreicher, 2016). Hermawati et al (2018) demonstrated the relationship between early electronic screen exposure and autistic-like behaviors (e.g., language delay, short attention span and hyperactivity) in children (Hermawati, Rahmadi, Sumekar, \& Winarni, 2018). Yurika et al. (2018) found that excessive screen time in children was associated with autistic symptoms (i.e., ocular problems, hyperactivity and language delay). A recent review of the literature on this area found that screen media itself is the causal factor for ASD (Slobodin, Heffler, \& Davidovitch, 2019). In a recent study, Heffler, Sienko, Subedi, McCann, and Bennett (2020) investigated association of early-life social and digital media experiences with development of autism spectrum disorder-like symptoms and found that television and/or video exposure and less caregiver-child interactive play at 12 months of age were each significantly associated with greater ASD-like symptoms, but not with the risk of ASD.

In some cases, digital devices replaced by child's active relationship with environment and children are expose to permanent digital devices. In this lifestyle that called "Digital Nanning", children lose the opportunity to interact with their mothers or caregivers and do not get rich environment that are necessary for normal development of the nervous system (Pouretemad., Sadeghi, \& Rahmati, 2017). Digital Nanning referred to the conditions that young children had been exposed to digital devices for more than half their waking hours (Sadeghi, Pouretemad, Khosrowabadi, Fathabadi, \& Nikbakht, 2019a).

Throughout this paper we use "Post Digital Nanning Autism Syndrome (PDNAS)" refer to condition where after young children excessive exposure to digital devices (for more than half their waking hours), develop subclinical autism symptoms that we hypothesized this is a new subtype of autistic spectrum disorder that caused by children lifestyle and excessive screen-time (Sadeghi, Pouretemad, Khosrowabadi, Fathabadi, \& Nikbakht, 2019c). In this case, it is assumed that young children autism symptoms caused by early excessive exposure to digital devices. Children with PDNAS have difficulties in eye contact and communication skills and also, they have restricted and repetitive behaviors or interests. At the moment, because of the similarity of PDNAS and formal autism, make differential diagnosis is impossible. Although, in clinical case observations we have found some differences in symptoms profiles of this two groups of children (such as more visual hypersensitivity and better joint attention in children with PDNAS rather the children with autism), further studies, will need to be performed about PDNAS. Similarly, Zamfir (2018) suggested that early media exposure represents a different type of autism, called "virtual autism" (Zamfir, 2018).

Despite the value of screens, it has now been suggested that excessive engagement with screen media may lead to deprivation and social isolation (Bickham \& Rich, 2006; Ray \& Jat, 2010; 
Stiglic \& Viner, 2019; Taheri, 2013). There is evidence that the restricted environments and experiences and early social isolation can cause autism symptoms and repetitive behaviors. For example, Rutter et al. $(1999,2007)$ reported that children who grew up in institutions are at increased risk for "quasi-autistic" patterns of behavior (Rutter et al., 1999; Rutter et al., 2007). In other study, Another Hoksbergen et al. (2005) suggested a similar disorder that described as post-institutional autistic syndrome (PIAS) in 16 percent of previously institutionalized infants (Hoksbergen, Ter Laak, Rijk, van Dijkum, \& Stoutjesdijk, 2005). Although children with PIAS and quasi-autism have overlapping symptoms, including social communication difficulties and repetitive behaviors with ASD, these researchers described several features that distinguish between children with PIAS and quasi-autism, which are different from those commonly found in " ordinary" ASD. For instance, equal Female: Male Ratio (1: 4 female to male ratio compared to formal autism) and the general tendency to reduce the severity of autism symptoms over time (Levin, Fox, Zeanah Jr, \& Nelson, 2015).

As mentioned above, much work on the early excessive screen time consequences has been carried out, however few studies have been performed to evaluated the interventions for children with autism symptoms and excessive screen-time (PDNAS). For example, Özyurt et al., (2018) used the Positive Parenting Program (Triple P) intervention and Adams et al (2018) used the responsive parenting intervention to show that parent training is effective method for reduce the duration of children's screen time and television exposure (Adams et al., 2018; Özyurt, Dinsever, Çalişkan, \& Evgin, 2018). Also, in our more recent studies, we demonstrated that early parentchild interaction is associate with normalized patterns of brain activity (EEG absolute, relative and ratio power), which were associated with reducing the duration of screen-time, improvements in executive functioning problems, autism symptoms and repetitive behaviors of young children with autism symptoms and excessive screen time (Sadeghi et al., 2019a; Sadeghi, Pouretemad, Khosrowabadi, Fathabadi, \& Nikbakht, 2019b; Sadeghi et al., 2019c). It is suggested that parents as primary caregivers can be considered the initial experts on their children, spending more time with them than almost any other adult the child encounters in their day-to-day lifetime (Bordelon \& Bradley, 2019). Although, it is important to note that parents of children with autism spectrum disorder symptoms themselves may need to interventions because of the high stress they experience (Anyanwu et al., 2019; Davis \& Carter, 2008; Gong et al., 2015; McStay, Dissanayake, Scheeren, Koot, \& Begeer, 2014; Pottie, Cohen, \& Ingram, 2009; Soltanifar et al., 2015) and involving these parents in interventions for their children can reduce their stress and enhance their mental health (Catalano, Holloway, \& Mpofu, 2018; Estes et al., 2014).

As described earlier, previous studies have revealed that parent training is the most effective approach to the reduce children's screen-time and make communicative lifestyle (Adams et al., 2018; Özyurt et al., 2018; Sadeghi et al., 2019a, 2019b, 2019c), only a small proportion of families benefit from evidence-based interventions (Jensen et al., 2011). The families problems to receiving center-based parent training include the difficulties of attending in intervention in terms of costs, time, and location and the lack of trained staff that can provide treatments (Kazdin \& Blase, 2011; Kazdin \& Rabbitt, 2013). Internet-based parent training interventions has a lot of benefits over traditional interventions, such as overcoming geographical barriers for parents to receive psychoeducation, higher fidelity, greater accessibility, convenience, and reduced time and costs (Aqdassi, Pouretemad, Fathabadi, \& Sadeghi, 2019; Barak \& Grohol, 2011; Breitenstein, Gross, \& Christophersen, 2014; Hammond et al., 2012; Khanna, Aschenbrand, \& Kendall, 2007; Ristkari et al., 2019). Internet-based interventions have already 
been used to treatment autism spectrum disorder symptoms and its effectiveness has been demonstrated (Ashburner, Vickerstaff, Beetge, \& Copley, 2016; Baharav \& Reiser, 2010; Boisvert, Lang, Andrianopoulos, \& Boscardin, 2010; Gibson, Pennington, Stenhoff, \& Hopper, 2010; Hamad, Serna, Morrison, \& Fleming, 2010; Lindgren et al., 2016; Shire, Worthman, Shih,

\& Kasari, 2020; Speyer et al., 2018; Sutherland, Trembath, \& Roberts, 2018; Vismara, McCormick, Young, Nadhan, \& Monlux, 2013). However, no studies exist on internet-based parent training efficacy for children with PDNAS and the aim of this study is to compare treatment outcomes of an internet-based intervention with a center-based intervention for young children with PDNAS.

\section{Materials and Method}

\subsection{Participants}

Participants were 40 dyads of parents and their children with PDNAS (aged between 18-48 months) who referred to Tehran Autism Center. All children had subclinical autism symptoms. The study participants were expose to digital devices for more than half their waking time and have subclinical autism symptoms that to describe this condition we used PDNAS term.

\subsection{Measures}

\subsubsection{Lifestyle Checklist}

To assess a child's lifestyle, we have compiled a checklist that parents had to record at home every 5 minutes what their child is doing. In this method, we measured the child's sleep, waking time, screen time, and interactions. Parents complete this checklist two days (one day a week and one day a weekend). Based on the results of this instrument, children with subthreshold autism symptoms who had been exposed to digital devices for more than half their waking hours are identified as children with PDNAS. We calculated the average hours of child's screen time. For more details, see our previous study (Sadeghi et al., 2019a, 2019b, 2019c).

\subsubsection{Repetitive Behavior Scale-Revised (RBS-R)}

RBS-R (Bodfish, Symons, Parker, \& Lewis, 2000) is a 43-item informant-based rating scale intended to assess 6 dimensions of repetitive behavior (Stereotyped Behavior, Self-Injurious Behavior, Compulsive Behavior, Ritualistic Behavior, Sameness Behavior, and Restricted Behavior). The sum of the ratings for all the items in a subscale gives the 'Overall Score'. Lam and Aman (2007) reported internal consistency of subscale from 0.78 to 0.91 and construct validity for subscale from 0.68 to 0.98 . The mentioned descriptions were mostly reproduced from our previous study using similar instrument (Sadeghi et al., 2019a). RRBs is one of the core symptoms of ASD and include a heterogeneous set of behaviors such as intense preoccupations, stereotyped movements, and resistance to change (American Psychiatric Association, 2013; Boyd, McDonough, \& Bodfish, 2012).

\subsubsection{Gilliam Autism Rating Scale-Second Edition (GARS-2)}

GARS-2 (Gilliam, 1995) used for assess severity of autism before and after the parent training intervention. 
GARS-2 is a 42- items, 4-point Likert scale that collects information on three areas of communication, stereotyped behaviors and social interaction. Its internal reliability ranges from 0.88 to 0.93 . The scale has high criterion validity with other tools, such as, the Autism Behavior Checklist (0.94) (Healy, O'Connor, Leader, \& Kenny, 2008).

\subsubsection{Parenting Stress Index-Short Form (PSI-SF)}

PSI-SF (Abidin, 1995) is a 36-item self-reporting scale that assess perceived stress related to parenting role. PSI is a widely used tool for measuring parental stress in parents of children with ASD (Hayes \& Watson, 2013). The PSI-SF consists of three 12-item subscales, which include the Parental Distress (PD), Parent-Child Dysfunctional Interaction (PCDI) and Difficult Child (DC) subscales, as well as a Total Stress scale. Previous studies demonstrated that all three subscales and the total stress scale have a good internal consistency, test-retest reliability, and validity (Haskett, Ahern, Ward, \& Allaire, 2006; Whiteside-Mansell et al., 2007).

\subsection{Intervention}

All parents trained to minimize their child's screen time and to have interactions with children. They received the parent training intervention (Emotional Diet Intervention) developed by Pouretemad (2010) and this intervention principle is based on Focused Playtime Intervention (FPI) (Siller, Hutman, \& Sigman, 2013). Our intervention consists of 7 parent training sessions (one session per week, 90 minutes per group session and 30 minutes per individually session). Session topics are described in Table 1. Parents in both groups received intervention in the same treatment modules, chronological order and time-frame

Table 1: Parent training sessions summery

\begin{tabular}{|c|c|c|c|}
\hline Objective & Session & Method & Activities examples \\
\hline 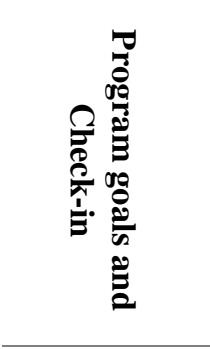 & One & Group & $\begin{array}{l}\text { - Introducing group members } \\
\text { Talk about excessive screen time } \\
\text { - Discuss the nature of this intervention } \\
\text { - Teaching parents to observe and record children's daily life activities } \\
\text { and routines } \\
\text { - Review the Videotape an episode of mother - child interaction } \\
\text { Help parent develop a profile of their child's symptoms and } \\
\text { communication skills }\end{array}$ \\
\hline \multirow{2}{*}{ 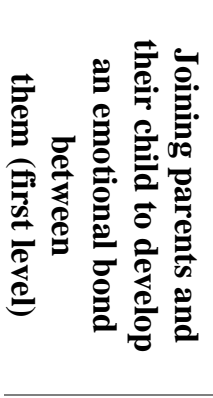 } & Two & Group & $\begin{array}{l}\text { - Training parents to minimize digital devices use and managing it } \\
\text { - Help parents manage child's routines (i.e., sleeping, feeding, etc.) } \\
\text { - Training parents in simple activities or plays for develop a relationship } \\
\text { with the child (e.g. physical play, emotional activities, and sensory paly) } \\
\text { - Help parents find shared activity } \\
\text { - Training parents to increase children's eye contact }\end{array}$ \\
\hline & Three & Individually & $\begin{array}{l}\text { - Review the videotape of previous session activities to identify problems } \\
\text { and discuss about issues. } \\
\text { - Discuss about parents' common problems in last week }\end{array}$ \\
\hline \multirow[t]{2}{*}{ 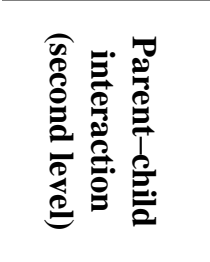 } & Four & Group & $\begin{array}{l}\text { - Training techniques for parents to enhance communication with children } \\
\text { while playing with toys } \\
\text { - Training parents to develop a special play time routine } \\
\text { Training parents to prevent (not confront) repetitive and non- } \\
\text { communicative activities }\end{array}$ \\
\hline & Five & Individually & - Review the videotape of previous session activities to identify problems \\
\hline
\end{tabular}


and discuss about these.

- Discuss about parents' common issues in last week

- Training parents to establish shared control over the activities during

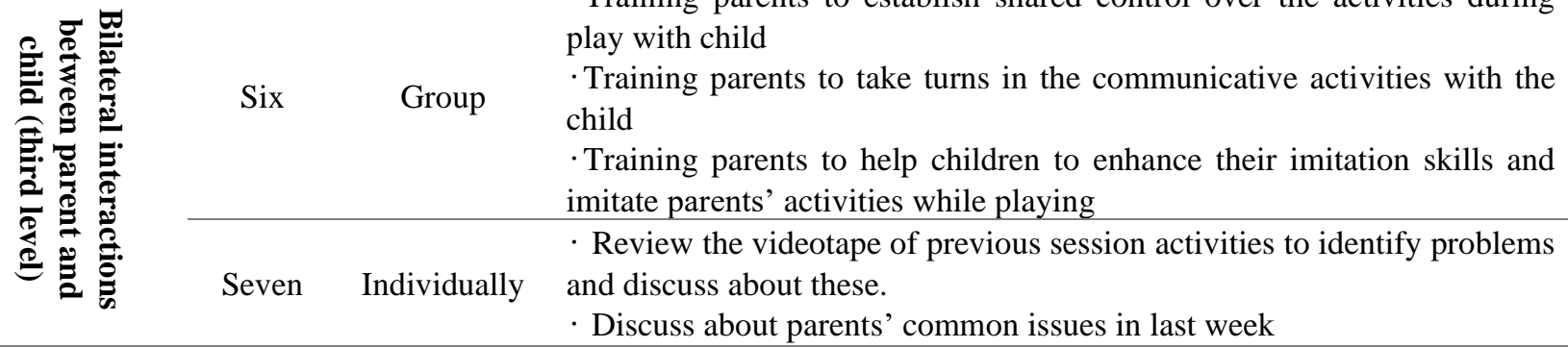

\subsection{Procedures}

Forty children with PDNAS aged 18-48 months and their parents that referred to Tehran Autism Center (Tehran, Iran) were recruit for this study. After receive written consent from the parents, who were residents of Tehran allocated in the center-based group and parents who were away from Tehran were placed in the internet-based group. Further, we didn't choose a witting list or control group for ethical considerations and small available sample size.

The therapist was an Iranian Ph.D. degree child clinical psychologist who was in Tehran. The center-based parent training was conducted on Tehran Autism Center and for internet-based parent training was conduct on Skype software video call (Figure 1) for five groups of parents (each group included the parents of 4 children). Parents only attended in both online and face-toface sessions without their children. Before and after the intervention (pre-test and post-test), the therapist sent the online link of questionnaires to the parents on both groups. Figure 2 shows the flowchart of the procedure of the study.

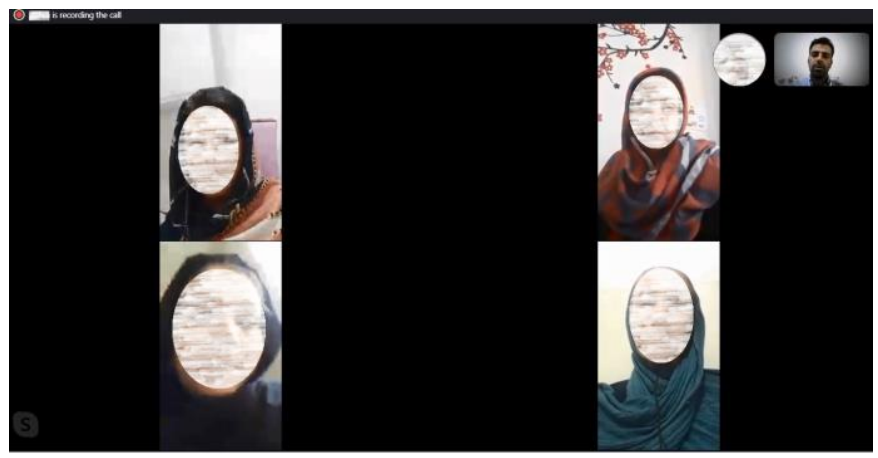

Figure 1. Internet-based intervention video calling sessions 


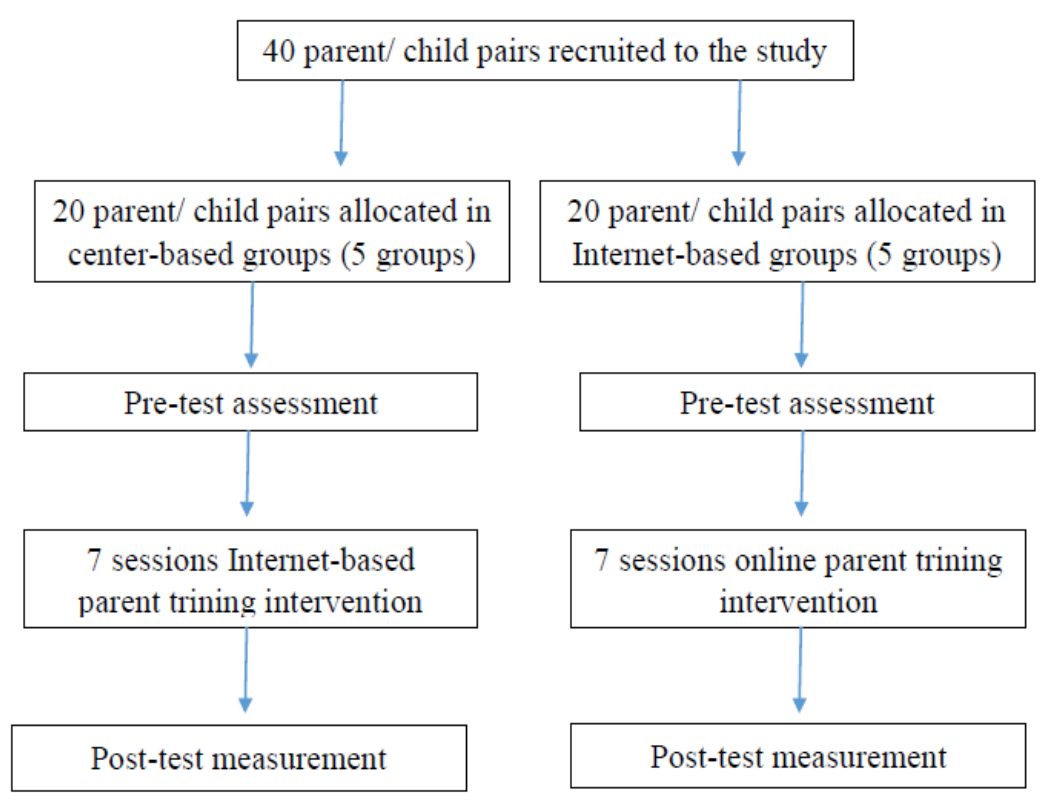

Figure 2. Flowchart of the procedure of the study

\subsection{Statistical analysis}

Independent t-test was conducted to test for differences in subjects' baseline characteristics. A multivariate generalized linear model (GLM) used to compare outcome measures between internet-based intervention and center-based intervention groups. Also, dependent t-test used to compare within groups effects. For improved interpretation and comparison, we calculated Cohen's $d$ and Eta effect sizes. The data were analyze using SPSS 22 software (Corp, 2013).

\section{Results}

In this study, 40 young children aged 18 to 48 months (mean= 31,17 months, $\mathrm{SD}=6.89$ ) participated. Baseline characteristics of the two groups included in this research are shown in Table 2. No significant differences were found between the internet-based and center-based intervention groups at baseline $(\mathrm{p}<0.05)$.

Table 2. Baseline characteristics of the center-based $(n=20)$ and internet-based $(n=20)$ groups

\begin{tabular}{|c|c|c|c|c|c|c|c|}
\hline Variable & & Group & $\underline{M}$ & $\underline{S D}$ & $\underline{M D}$ & $t$ & $\boldsymbol{P}$ \\
\hline \multirow{2}{*}{ Age } & \multirow{2}{*}{ Month } & CBG & $\overline{31} .90$ & $\overline{7.90}$ & \multirow{2}{*}{1.45} & \multirow{2}{*}{0.66} & \multirow{2}{*}{0.53} \\
\hline & & IBG & 30.45 & 5.83 & & & \\
\hline \multirow{8}{*}{ 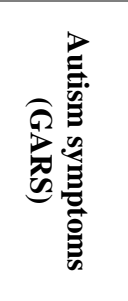 } & \multirow{2}{*}{ Stereotyped behaviors } & CBG & 39.15 & 5.88 & \multirow{2}{*}{3.48} & \multirow{2}{*}{1.95} & \multirow{2}{*}{0.06} \\
\hline & & IBG & 35.67 & 5.54 & & & \\
\hline & \multirow{2}{*}{ Communication } & $\mathrm{CBG}$ & 42.35 & 5.85 & \multirow{2}{*}{-0.94} & \multirow{2}{*}{-0.42} & \multirow{2}{*}{0.67} \\
\hline & & IBG & 43.29 & 8.06 & & & \\
\hline & \multirow{2}{*}{ Social interaction } & CBG & 38.60 & 6.34 & \multirow{2}{*}{-0.21} & \multirow{2}{*}{-0.11} & \multirow{2}{*}{0.92} \\
\hline & & IBG & 38.91 & 6.31 & & & \\
\hline & \multirow{2}{*}{ Autism Index (total score) } & CBG & 120.10 & 14.60 & \multirow{2}{*}{2.34} & \multirow{2}{*}{0.49} & \multirow{2}{*}{0.62} \\
\hline & & IBG & 117.76 & 15.59 & & & \\
\hline $\bar{\sigma} \bar{\theta} \odot \Xi$ & Stereotyped behaviors & $\mathrm{CBG}$ & 11.10 & 2.73 & 0.29 & 0.33 & 0.74 \\
\hline
\end{tabular}




\begin{tabular}{|c|c|c|c|c|c|c|c|}
\hline & & IBG & 10.81 & 2.86 & \multirow{3}{*}{0.55} & \multirow{3}{*}{0.95} & \multirow{3}{*}{0.35} \\
\hline & \multirow{2}{*}{ Self-injurious behaviors } & CBG & 9.65 & 2.21 & & & \\
\hline & & IBG & 9.01 & 1.48 & & & \\
\hline & \multirow{2}{*}{ Ritualistic behaviors } & CBG & 10.75 & 3.17 & \multirow{2}{*}{0.80} & \multirow{2}{*}{0.89} & \multirow{2}{*}{0.38} \\
\hline & & IBG & 9.52 & 2.54 & & & \\
\hline & \multirow{2}{*}{ Sameness behaviors } & CBG & 9.10 & 3.24 & \multirow{2}{*}{-0.38} & \multirow{2}{*}{-0.38} & \multirow{2}{*}{0.70} \\
\hline & & IBG & 9.48 & 3.03 & & & \\
\hline & \multirow{2}{*}{ Restricted behaviors } & CBG & 17.10 & 4.83 & \multirow{2}{*}{1} & \multirow{2}{*}{0.74} & \multirow{2}{*}{0.47} \\
\hline & & IBG & 16.10 & 3.87 & & & \\
\hline & \multirow{2}{*}{ Compulsive behaviors } & CBG & 8.15 & 3.25 & \multirow{2}{*}{1.29} & \multirow{2}{*}{1.40} & \multirow{2}{*}{0.17} \\
\hline & & IBG & 6.86 & 2.65 & & & \\
\hline & \multirow{2}{*}{ Total score } & CBG & 65.85 & 13.77 & \multirow[b]{2}{*}{3.56} & \multirow[b]{2}{*}{0.87} & \multirow{2}{*}{0.39} \\
\hline & & IBG & 62.29 & 12.39 & & & \\
\hline \multirow{8}{*}{ 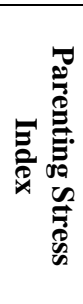 } & \multirow{2}{*}{ Parental distress } & CBG & 34.45 & 9.26 & \multirow{2}{*}{-1.35} & \multirow{2}{*}{-0.53} & \multirow{2}{*}{0.60} \\
\hline & & IBG & 33.40 & 7 & & & \\
\hline & & $\mathrm{CBG}$ & 33.50 & 7.59 & & & \\
\hline & Parent-child dysfunctional interaction & IBG & 33.25 & 5.85 & -0.50 & -0.24 & 0.81 \\
\hline & & $\mathrm{CBG}$ & 33.65 & 9.30 & & & \\
\hline & Difficult child & IBG & 34 & 8.06 & 0.54 & 0.20 & 0.84 \\
\hline & & $\mathrm{CBG}$ & 101.60 & 20.06 & & & \\
\hline & Total parentıng stress score & IBG & 100.65 & 16.50 & -1.31 & -0.23 & 0.82 \\
\hline
\end{tabular}

Abbreviations: CBG; center-based group, IBG; Internet-based group, $\underline{\mathrm{M}}$; mean, $\underline{\mathrm{SD}}$; standard deviation, $\underline{\mathrm{MD}}$; mean differences.

In the analyses presented in Table 3, results show statistically significant change in both groups from the beginning of treatment to the end of treatment. Intervention was effective for decreasing autism symptoms $(p<0.0001)$, repetitive behaviors $(p<0.001)$, and parenting stress $(p<0.05)$ in internet-based group. Also, intervention was effective for decreasing autism symptoms $(\mathrm{p}<0.0001)$, repetitive behaviors $(\mathrm{p}<0.0001)$, and parenting stress $(\mathrm{p}<0.0001)$ in center-based group. Effect sizes in the internet-based group and the center-based group can be considered large for autism and repetitive behaviors and medium for parenting stress (Cohen, 2013).

Table 3. Intervention effect on outcome measures per intervention condition (within subject)

\begin{tabular}{|c|c|c|c|c|c|c|c|c|c|c|c|c|}
\hline \multirow{2}{*}{\multicolumn{2}{|c|}{ Variable }} & \multicolumn{6}{|c|}{ Internet-based intervention group } & \multicolumn{5}{|c|}{ Centre-based intervention group } \\
\hline & & Time & $\underline{M}$ & $\underline{S D}$ & $t$ & Sig & d & $M$ & $\underline{S D}$ & $t$ & Sig & d \\
\hline \multirow{3}{*}{\multicolumn{2}{|c|}{ 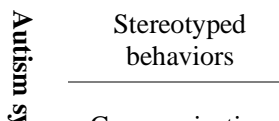 }} & Pre-test & 35.67 & 5.5 & \multirow{2}{*}{1.78} & \multirow{2}{*}{0.09} & \multirow{2}{*}{0.39} & 39.15 & 5.88 & \multirow{2}{*}{3.79} & \multirow{2}{*}{0.001} & \multirow{2}{*}{0.98} \\
\hline & & Post-test & 33.52 & 5.58 & & & & 34.30 & 4.24 & & & \\
\hline & & Pre-test & 43.29 & 8.057 & \multirow{2}{*}{3.25} & \multirow{2}{*}{0.004} & \multirow{2}{*}{0.70} & 42.35 & 5.85 & \multirow{2}{*}{3.11} & \multirow{2}{*}{0.006} & \multirow{2}{*}{0.80} \\
\hline E्छ & Communication & Post-test & 38 & 6.92 & & & & 37.55 & 6.19 & & & \\
\hline $\mathrm{E}$ & \multirow{2}{*}{$\begin{array}{l}\text { Social } \\
\text { interaction }\end{array}$} & Pre-test & 38.81 & 6.30 & \multirow{2}{*}{4.82} & \multirow{2}{*}{0.0001} & \multirow{2}{*}{0.86} & 38.60 & 6.34 & \multirow{2}{*}{4.17} & \multirow{2}{*}{0.001} & \multirow{2}{*}{1.05} \\
\hline â & & Post-test & 33.14 & 6.83 & & & & 32.95 & 4.19 & & & \\
\hline $\mathbb{\pi}_{\pi}$ & \multirow{2}{*}{$\begin{array}{l}\text { Autism Index } \\
\text { (total score) }\end{array}$} & Pre-test & 117.76 & 15.59 & \multirow{2}{*}{4.85} & \multirow{2}{*}{0.0001} & \multirow{2}{*}{0.81} & 120.10 & 14.60 & \multirow{2}{*}{4.52} & \multirow{2}{*}{0.0001} & \multirow{2}{*}{1.11} \\
\hline & & Post-test & 104.67 & 16.84 & & & & 104.80 & 12.82 & & & \\
\hline
\end{tabular}




\begin{tabular}{|c|c|c|}
\hline \multirow{14}{*}{ 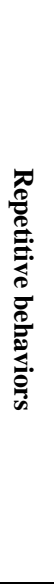 } & \multirow{2}{*}{$\begin{array}{c}\text { Stereotyped } \\
\text { behaviors }\end{array}$} & Pre-test \\
\hline & & Post-test \\
\hline & \multirow{2}{*}{$\begin{array}{l}\text { Self-injurious } \\
\text { behaviors }\end{array}$} & Pre-test \\
\hline & & Post-test \\
\hline & \multirow{2}{*}{$\begin{array}{l}\text { Ritualistic } \\
\text { behaviors }\end{array}$} & Pre-test \\
\hline & & Post-test \\
\hline & \multirow{2}{*}{$\begin{array}{l}\text { Sameness } \\
\text { behaviors }\end{array}$} & Pre-test \\
\hline & & Post-test \\
\hline & \multirow{2}{*}{$\begin{array}{l}\text { Restricted } \\
\text { behaviors }\end{array}$} & Pre-test \\
\hline & & Post-test \\
\hline & \multirow{2}{*}{$\begin{array}{l}\text { Compulsive } \\
\text { behaviors }\end{array}$} & Pre-test \\
\hline & & Post-test \\
\hline & \multirow{2}{*}{ Total score } & Pre-test \\
\hline & & Post-test \\
\hline \multirow{8}{*}{ 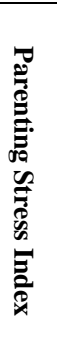 } & \multirow{2}{*}{ Parental distress } & Pre-test \\
\hline & & Post-test \\
\hline & \multirow{2}{*}{$\begin{array}{l}\text { Parent-child } \\
\text { dysfunctional } \\
\text { interaction }\end{array}$} & Pre-test \\
\hline & & Post-test \\
\hline & \multirow{2}{*}{ Difficult child } & Pre-test \\
\hline & & Post-test \\
\hline & \multirow{2}{*}{$\begin{array}{l}\text { Total parenting } \\
\text { stress score }\end{array}$} & Pre-test \\
\hline & & Post-test \\
\hline
\end{tabular}

\begin{tabular}{|c|c|c|c|c|}
\hline 10.81 & 2.86 & \multirow{2}{*}{5.25} & \multirow{2}{*}{0.0001} & \multirow{2}{*}{0.86} \\
\hline 8.62 & 2.18 & & & \\
\hline 9.09 & 1.48 & \multirow{2}{*}{1.63} & \multirow{2}{*}{0.12} & \multirow{2}{*}{0.32} \\
\hline 8.67 & 1.15 & & & \\
\hline 9.95 & 2.54 & \multirow{2}{*}{3.24} & \multirow{2}{*}{0.004} & \multirow{2}{*}{0.48} \\
\hline 8.95 & 1.53 & & & \\
\hline 9.48 & 3.03 & \multirow{2}{*}{2.47} & \multirow{2}{*}{0.023} & \multirow{2}{*}{0.64} \\
\hline 7.76 & 2.32 & & & \\
\hline 16.09 & 3.87 & \multirow{2}{*}{2.43} & \multirow{2}{*}{0.025} & \multirow{2}{*}{0.65} \\
\hline 13.81 & 3.04 & & & \\
\hline 6.86 & 2.65 & \multirow{2}{*}{2} & \multirow{2}{*}{0.059} & \multirow{2}{*}{0.44} \\
\hline 5.81 & 2.09 & & & \\
\hline 62.29 & 12.39 & \multirow{2}{*}{3.88} & \multirow{2}{*}{0.001} & \multirow{2}{*}{0.76} \\
\hline 53.62 & 10.25 & & & \\
\hline 33.40 & 7.04 & \multirow{2}{*}{1.42} & \multirow{2}{*}{0.17} & \multirow{2}{*}{0.34} \\
\hline 30.25 & 10.99 & & & \\
\hline 33.25 & 5.88 & \multirow[b]{2}{*}{3.11} & \multirow[b]{2}{*}{0.006} & \multirow[b]{2}{*}{0.71} \\
\hline 28.85 & 6.49 & & & \\
\hline 34 & 8.22 & \multirow{2}{*}{2.27} & \multirow{2}{*}{0.035} & \multirow{2}{*}{0.50} \\
\hline 29.80 & 8.51 & & & \\
\hline 100.65 & 16.85 & \multirow{2}{*}{2.74} & \multirow{2}{*}{0.013} & \multirow{2}{*}{0.55} \\
\hline 89.45 & 23.06 & & & \\
\hline
\end{tabular}

\begin{tabular}{|c|c|c|c|c|}
\hline 11.10 & 2.73 & \multirow{2}{*}{3.67} & \multirow{2}{*}{0.002} & \multirow{2}{*}{1.09} \\
\hline 8.70 & 1.49 & & & \\
\hline 9.65 & 2.20 & \multirow{2}{*}{2.21} & \multirow{2}{*}{0.04} & \multirow{2}{*}{0.59} \\
\hline 8.65 & 0.99 & & & \\
\hline 10.75 & 3.18 & \multirow{2}{*}{1.64} & \multirow{2}{*}{0.12} & \multirow{2}{*}{0.36} \\
\hline 9.80 & 1.99 & & & \\
\hline 9.10 & 3.24 & \multirow{2}{*}{1.68} & \multirow{2}{*}{0.11} & \multirow{2}{*}{0.38} \\
\hline 8.05 & 2.095 & & & \\
\hline 17.10 & 4.83 & \multirow{2}{*}{4.52} & \multirow{2}{*}{0.0001} & \multirow{2}{*}{0.91} \\
\hline 13.70 & 2.10 & & & \\
\hline 8.15 & 3.25 & \multirow{2}{*}{3.49} & \multirow{2}{*}{0.002} & \multirow{2}{*}{1.05} \\
\hline 5.55 & 1.32 & & & \\
\hline 65.85 & 13.77 & \multirow{2}{*}{4.51} & \multirow{2}{*}{0.0001} & \multirow{2}{*}{1.07} \\
\hline 54.45 & 5.89 & & & \\
\hline 34.45 & 9.26 & \multirow{2}{*}{1.90} & \multirow{2}{*}{0.07} & \multirow{2}{*}{0.38} \\
\hline 31 & 8.66 & & & \\
\hline 33.50 & 7.59 & \multirow[b]{2}{*}{3.42} & \multirow[b]{2}{*}{0.003} & \multirow[b]{2}{*}{0.45} \\
\hline 29.95 & 7.35 & & & \\
\hline 33.65 & 9.30 & \multirow{2}{*}{2.49} & \multirow{2}{*}{0.02} & \multirow{2}{*}{0.47} \\
\hline 29.40 & 8.82 & & & \\
\hline 101.60 & 20.06 & \multirow{2}{*}{3.13} & \multirow{2}{*}{0.004} & \multirow{2}{*}{0.53} \\
\hline 90.75 & 21.03 & & & \\
\hline
\end{tabular}

Abbreviations: CBG; center-based group, IBG; Internet-based group, $\underline{\mathrm{M}}$; mean, $\underline{\mathrm{SD}}$; standard deviation, $\underline{d}$; Cohen's d effect size, MD; mean differences.

Between groups analyses for compare the effects of intervention between two groups, shown in Table 4, do not reveal a significant between groups difference $(\mathrm{p}<0.05)$.

Table 4. Tests of between-subjects' effects to compare two groups in outcome variables

\begin{tabular}{|c|c|c|c|c|c|}
\hline \multicolumn{2}{|l|}{ Variable } & $\underline{S S}$ & $\boldsymbol{F}$ & $\underline{\text { Sig }}$ & $\eta 2$ \\
\hline \multirow{4}{*}{$\begin{array}{l}\text { Autism } \\
\text { symptoms } \\
\text { (GARS) }\end{array}$} & Stereotyped behaviors & 1.062 & 0.055 & 0.817 & 0.002 \\
\hline & Communication & 4.123 & 0.111 & 0.742 & 0.004 \\
\hline & Social interaction & 0.522 & 0.020 & 0.889 & 0.001 \\
\hline & Autism Index (total score) & 14.316 & 0.079 & 0.781 & 0.003 \\
\hline \multirow{7}{*}{$\begin{array}{l}\text { Repetitive } \\
\text { behaviors }\end{array}$} & Stereotyped behaviors & 0.103 & 0.037 & 0.849 & 0.001 \\
\hline & Self-injurious behaviors & 0.032 & 0.036 & 0.852 & 0.001 \\
\hline & Ritualistic behaviors & 0.222 & 0.127 & 0.724 & 0.005 \\
\hline & Sameness behaviors & 0.129 & 0.032 & 0.860 & 0.001 \\
\hline & Restricted behaviors & 5.957 & 0.958 & 0.337 & 0.037 \\
\hline & Compulsive behaviors & 4.399 & 1.339 & 0.258 & 0.051 \\
\hline & Total score & 10.299 & 0.180 & 0.675 & 0.007 \\
\hline \multirow{4}{*}{$\begin{array}{c}\text { Parenting Stress } \\
\text { Index }\end{array}$} & Parental distress & 78.639 & 1.188 & 0.286 & 0.045 \\
\hline & Parent-child dysfunctional interaction & 0.566 & 0.017 & 0.898 & 0.001 \\
\hline & Difficult child & 4.496 & 0.087 & 0.770 & 0.003 \\
\hline & Total parenting stress score & 56.250 & 0.188 & 0.668 & 0.007 \\
\hline
\end{tabular}

Abbreviations: $\underline{\boldsymbol{S}}$; Sum of Squares, $\boldsymbol{\eta}^{2}$ : Eta effect size

In overall, both internet-based and center-based participants showed reductions in autism symptoms, repetitive behaviors and parenting stress, with no significant differences by treatment modality.

In addition, to investigate the relationship between the severity of the children's autism symptoms (Figure 3), repetitive behaviors (Figure 4) and their parents' reports of stress, 
Pearson's correlations were carried out between the parenting stress total score, children's repetitive behaviors total score and autism symptoms total score measures in the all participants. The parenting stress showed a statistically significant correlation with children's autism symptoms $(\mathrm{r}=0.53, p<0.0001)$. Furthermore, the parenting stress showed a statistically significant correlation with the severity of children's repetitive behaviors $(\mathrm{r}=0.61, p<0.0001)$.

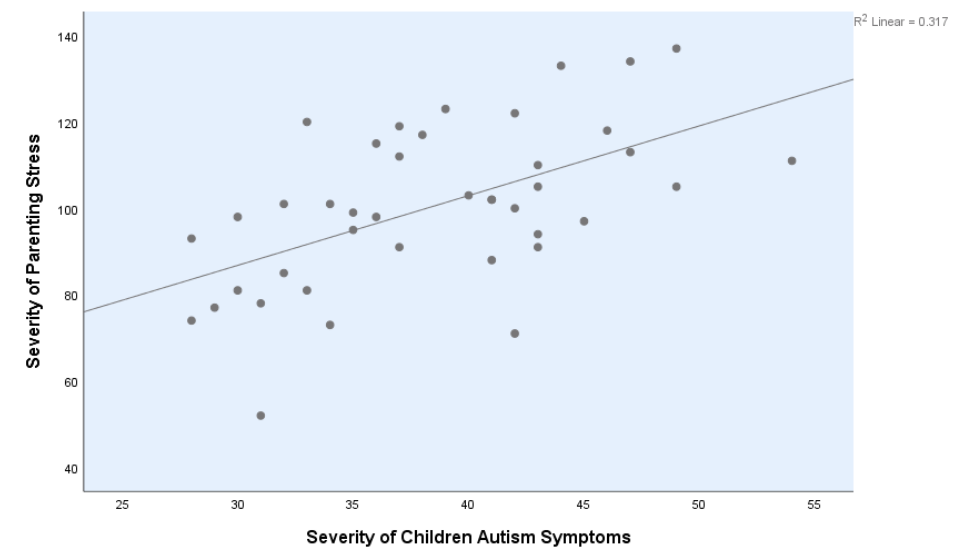

Figure 3. Parenting stress in relation to children's autism symptoms severity

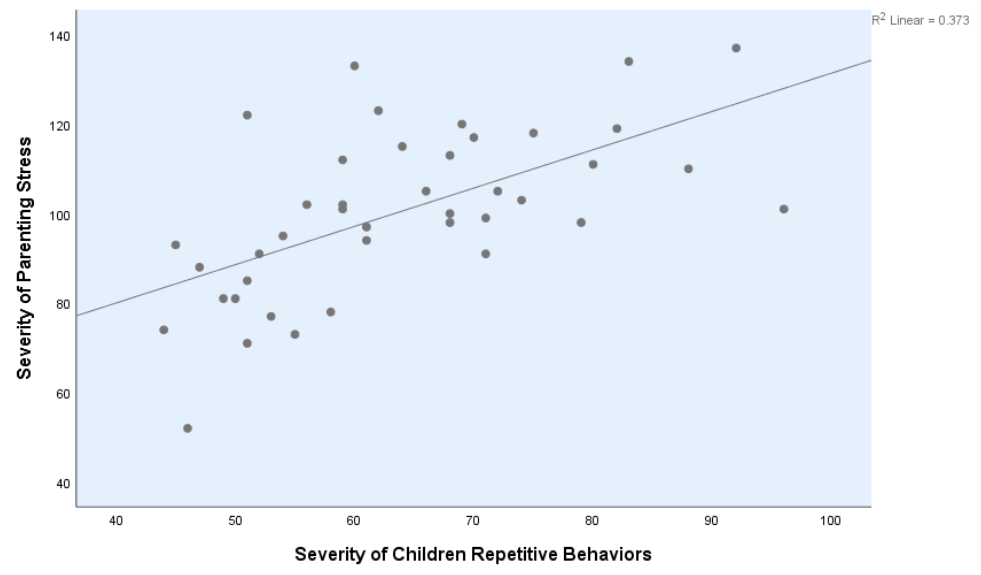

Figure 4. Parenting stress in relation to children's repetitive behaviors severity

\section{Discussion}

The aim of this study was to test an internet-based parent training intervention against a comparable, traditional center-based parent training intervention for children with PDNAS. To our knowledge this is the first study for children with PDNAS comparing both intervention forms with equivalent treatment modules and treatment length. The main finding of this study is that the internet-based parent training intervention is indeed as effective as center-based parent training intervention for children with PDNAS. This is in line with previous studies comparing face-to-face interventions with internet-based interventions for children with autism spectrum 
disorder (Ashburner et al., 2016; Baharav \& Reiser, 2010; Boisvert et al., 2010; Gibson et al., 2010; Hamad et al., 2010; Lindgren et al., 2016; Shire et al., 2020; Sutherland et al., 2018; Vismara et al., 2013). The within group effect size in the internet- based group ranged from $d=0.32$ to $d=0.86$ and in the center-based group ranged from $d=0.38$ to $d=1.09$ for autism symptoms, repetitive behaviors and parental stress.

The results of our study add to the growing empirical evidence for the efficacy of telepractice service-delivery models. It seems that by providing services to families over the Internet, many of the challenges and barriers to center-based service delivery (e.g., the traveling expenses, the cost of service delivery, the amount of travel time required of the provider and complications with scheduling center visits) may be reduced.

A possible explanation for the internet-based parent training effects on children's autism symptoms, repetitive behaviors and parent's stress is that this intervention can increase the number of intervention hours a child receives and improve child outcomes. In other words, parents as primary caregivers can be considered the initial experts on their children, spending more time with them online parent training makes it possible for the child to receive intervention with parents. Indeed, internet-based parent training is an interactive intervention that educate parents to have an intensive interaction with their child and promote they social-communication skills during play and daily routines. Also, one of the most effective strategies to treat autism symptoms such as repetitive behaviors at an early age is antecedent-based approaches (Boyd et al., 2012). In this study, parents were train to do proactive interactions and use some of the antecedent-based environmental modifications to remove any time slot for the incidence of noncommunicative behaviors and digital device use; as a result, the child was less likely to engage in media use and autistic behaviors and interests. Similarly, Zamfir (2018) in a longitudinal study observed better therapeutic outcomes for children with ASD with a history of excessive screen time before the age of three (more than 4 hours per-day) compared to children with ASD without a history of excessive screen time (Zamfir, 2018).

Further, the parental stress showed a significant correlation with children's autism symptoms and repetitive behaviors. This finding is consistent with the prior literature (Davis \& Carter, 2008; Gong et al., 2015; McStay et al., 2014; Pottie et al., 2009; Soltanifar et al., 2015). This result suggests that parents who perceive the autism symptoms and repetitive behaviors of their children are more severe tend to perceive more stress. A possible explanation for this might be that the demands of managing a child's autism symptoms and repetitive behaviors may threaten a parent's coping resources, resulting in greater stress and concern. In other word, the severity of child's autism symptoms and problems leads to more parenting stress, which in turn interferes with appropriate parenting strategies and results in more child behavior problems However, these explanations are speculations and require future studies and empirical foundation.

\section{Limitation}

Our study has a number of limitations, which need to be addressed. First, all measures administered as self-rated questionnaires in an online setting. Second, because we had not follow-up assessments, we cannot draw any conclusions about the long-term effects of the two interventions. Third, because of ethical considerations, we didn't choose a control group that these issues may have effects on our finding's validity. 


\section{Conclusion}

This study gives preliminary results that a brief internet-based parent training intervention for Children with PDNAS is as effective as comparable center-based parent training intervention. Internet-based intervention may be the solution for tackling children's autism symptoms, repetitive behaviors and parental stress in a more cost-effective way than traditional center-based interventions. It seems that internet-based interventions, has the potential to replace or augment traditional service models as center-based interventions to increase access to well-documented interventions. These interventions can reduce the cost of referring to health centers and rehabilitation, saving time, increasing access remote areas, and provide health care and the ability to receive long-term services in the natural context of the living environment to provide affordable services for all those in need. However, further study is needed to replicate these results and possible differences in underlying mechanisms between internet-based and centerbased interventions need to be evaluated.

\section{Declaration of Conflicting Interests}

The author(s) declared no potential conflicts of interest with respect to the research, authorship, and/or publication of this article.

\section{Funding}

The authors received no financial support for the study and publication of this article.

\section{References}

Abidin, R. R. (1995). Parenting stress index: Professional manual: Psychological Assessment Resources.

Adams, E. L., Marini, M. E., Stokes, J., Birch, L. L., Paul, I. M., \& Savage, J. S. (2018). INSIGHT responsive parenting intervention reduces infant's screen time and television exposure. international journal of behavioral nutrition and physical activity, 15(1), 24.

American Psychiatric Association. (2013). Diagnostic and statistical manual of mental disorders (DSM-5). Washington, DC: American Psychiatric Association.

Anyanwu, J. I., Onuigbo, L. N., Obiyo, N. O., Eze, U. N., Akaneme, I. N., Aye, E. N., . . Ezenwaji, I. O. (2019). Parenting Stress in Families of Children With Autism Spectrum Disorder: The Roles of the Extended Family. Global Journal of Health Science, 11(8).

Aqdassi, L., Pouretemad, H. R., Fathabadi, J., \& Sadeghi, S. (2019). Family-based tele-intervention of SPARK program on motor proficiency and severity of autism symptoms in children with high functioning autism spectrum disorder: A pilot study. Razi Journal of Medical Sciences, 25(11), 6171.

Ashburner, J., Vickerstaff, S., Beetge, J., \& Copley, J. (2016). Remote versus face-to-face delivery of early intervention programs for children with autism spectrum disorders: Perceptions of rural families and service providers. Research in Autism Spectrum Disorders, 23, 1-14.

Baharav, E., \& Reiser, C. (2010). Using telepractice in parent training in early autism. Telemedicine and eHealth, 16(6), 727-731.

Barak, A., \& Grohol, J. M. (2011). Current and future trends in internet-supported mental health interventions. Journal of Technology in Human Services, 29(3), 155-196.

Bickham, D. S., \& Rich, M. (2006). Is television viewing associated with social isolation?: roles of exposure time, viewing context, and violent content. Archives of pediatrics \& adolescent medicine, 160(4), 387-392. 
Bodfish, J. W., Symons, F. J., Parker, D. E., \& Lewis, M. H. (2000). Varieties of repetitive behavior in autism: Comparisons to mental retardation. Journal of Autism and Developmental Disorders, 30(3), 237-243.

Boisvert, M., Lang, R., Andrianopoulos, M., \& Boscardin, M. L. (2010). Telepractice in the assessment and treatment of individuals with autism spectrum disorders: A systematic review. Developmental neurorehabilitation, 13(6), 423-432.

Bordelon, A. E., \& Bradley, R. L. (2019). Family Inclusion. In Handbook of Interdisciplinary Treatments for Autism Spectrum Disorder (pp. 445-463): Springer.

Boyd, B. A., McDonough, S. G., \& Bodfish, J. W. (2012). Evidence-based behavioral interventions for repetitive behaviors in autism. Journal of Autism and Developmental Disorders, 42(6), 12361248.

Breitenstein, S. M., Gross, D., \& Christophersen, R. (2014). Digital delivery methods of parenting training interventions: a systematic review. Worldviews on Evidence - Based Nursing, 11(3), 168-176.

Catalano, D., Holloway, L., \& Mpofu, E. (2018). Mental health interventions for parent carers of children with autistic spectrum disorder: Practice guidelines from a Critical Interpretive Synthesis (CIS) systematic review. International journal of environmental research and public health, 15(2), 341.

Chang, H. Y., Park, E.-J., Yoo, H.-J., won Lee, J., \& Shin, Y. (2018). Electronic Media Exposure and Use among Toddlers. Psychiatry investigation.

Cohen, J. (2013). Statistical power analysis for the behavioral sciences: Academic press.

Corp, I. (2013). IBM SPSS statistics for windows, version 22.0. Armonk, NY: IBM Corp.

Davis, N. O., \& Carter, A. S. (2008). Parenting stress in mothers and fathers of toddlers with autism spectrum disorders: Associations with child characteristics. Journal of Autism and Developmental Disorders, 38(7), 1278.

Estes, A., Vismara, L., Mercado, C., Fitzpatrick, A., Elder, L., Greenson, J., . . . Young, G. (2014). The impact of parent-delivered intervention on parents of very young children with autism. Journal of Autism and Developmental Disorders, 44(2), 353-365.

Gibson, J. L., Pennington, R. C., Stenhoff, D. M., \& Hopper, J. S. (2010). Using desktop videoconferencing to deliver interventions to a preschool student with autism. Topics in Early Childhood Special Education, 29(4), 214-225.

Gilliam, J. E. (1995). Gilliam autism rating scale: Examiner's manual: Pro-ed.

Gong, Y., Du, Y., Li, H., Zhang, X., An, Y., \& Wu, B.-L. (2015). Parenting stress and affective symptoms in parents of autistic children. Science China Life Sciences, 58(10), 1036-1043.

Hamad, C. D., Serna, R. W., Morrison, L., \& Fleming, R. (2010). Extending the reach of early intervention training for practitioners: A preliminary investigation of an online curriculum for teaching behavioral intervention knowledge in autism to families and service providers. Infants and Young Children, 23(3), 195.

Hammond, G. C., Croudace, T. J., Radhakrishnan, M., Lafortune, L., Watson, A., McMillan-Shields, F., \& Jones, P. B. (2012). Comparative effectiveness of cognitive therapies delivered face-to-face or over the telephone: an observational study using propensity methods. PloS one, 7(9), e42916.

Haskett, M. E., Ahern, L. S., Ward, C. S., \& Allaire, J. C. (2006). Factor structure and validity of the parenting stress index-short form. Journal of Clinical Child \& Adolescent Psychology, 35(2), 302312.

Hayes, S. A., \& Watson, S. L. (2013). The impact of parenting stress: A meta-analysis of studies comparing the experience of parenting stress in parents of children with and without autism spectrum disorder. Journal of Autism and Developmental Disorders, 43(3), 629-642.

Healy, O., O'Connor, J., Leader, G., \& Kenny, N. (2008). Three years of intensive applied behavior analysis: A case study. Journal of Early and Intensive Behavior Intervention, 5(1), 4. 
Heffler, K. F., \& Oestreicher, L. M. (2016). Causation model of autism: Audiovisual brain specialization in infancy competes with social brain networks. Medical hypotheses, 91, 114-122.

Heffler, K. F., Sienko, D. M., Subedi, K., McCann, K. A., \& Bennett, D. S. (2020). Association of Early-Life Social and Digital Media Experiences With Development of Autism Spectrum Disorder-Like Symptoms. JAMA pediatrics.

Hermawati, D., Rahmadi, F. A., Sumekar, T. A., \& Winarni, T. I. (2018). Early electronic screen exposure and autistic-like symptoms. Intractable \& rare diseases research, 7(1), 69-71.

Hoksbergen, R., Ter Laak, J., Rijk, K., van Dijkum, C., \& Stoutjesdijk, F. (2005). Post-institutional autistic syndrome in Romanian adoptees. Journal of Autism and Developmental Disorders, 35(5), 615623.

Jensen, P. S., Goldman, E., Offord, D., Costello, E. J., Friedman, R., Huff, B., . . . Bird, H. (2011). Overlooked and underserved:"Action signs" for identifying children with unmet mental health needs. Pediatrics, 128(5), 970-979.

Kabali, H. K., Irigoyen, M. M., Nunez-Davis, R., Budacki, J. G., Mohanty, S. H., Leister, K. P., \& Bonner, R. L. (2015). Exposure and use of mobile media devices by young children. Pediatrics, peds. 20152151.

Kazdin, A. E., \& Blase, S. L. (2011). Rebooting psychotherapy research and practice to reduce the burden of mental illness. Perspectives on psychological science, 6(1), 21-37.

Kazdin, A. E., \& Rabbitt, S. M. (2013). Novel models for delivering mental health services and reducing the burdens of mental illness. Clinical Psychological Science, 1(2), 170-191.

Khanna, M., Aschenbrand, S. G., \& Kendall, P. C. (2007). New frontiers: Computer technology in the treatment of anxious youth. The Behavior Therapist.

Lam, K. S., \& Aman, M. G. (2007). The Repetitive Behavior Scale-Revised: independent validation in individuals with autism spectrum disorders. Journal of autism and developmental disorders, 37(5), 855-866.

Levin, A. R., Fox, N. A., Zeanah Jr, C. H., \& Nelson, C. A. (2015). Social communication difficulties and autism in previously institutionalized children. Journal of the American Academy of Child \& Adolescent Psychiatry, 54(2), 108-115. e101.

Lindgren, S., Wacker, D., Suess, A., Schieltz, K., Pelzel, K., Kopelman, T., . . . Waldron, D. (2016). Telehealth and autism: Treating challenging behavior at lower cost. Pediatrics, 137(Supplement 2), S167-S175.

McStay, R. L., Dissanayake, C., Scheeren, A., Koot, H. M., \& Begeer, S. (2014). Parenting stress and autism: The role of age, autism severity, quality of life and problem behaviour of children and adolescents with autism. Autism, 18(5), 502-510.

Özyurt, G., Dinsever, Ç., Çalişkan, Z., \& Evgin, D. (2018). Effects of triple P on digital technological device use in preschool children. Journal of Child and Family Studies, 27(1), 280-289.

Pottie, C. G., Cohen, J., \& Ingram, K. M. (2009). Parenting a child with autism: Contextual factors associated with enhanced daily parental mood. Journal of Pediatric Psychology, 34(4), 419-429.

Pouretemad, H. (2010). The role of digital nursing in shaping of autistic disorders symptoms. Paper presented at the The 3rd Child and Adolescent Psychiatry Conference, Tehran. .

Pouretemad., H. R., Sadeghi, S., \& Rahmati, Y. (2017). Digital Nanning and Autism Spectrum Disorder. Journal of Exceptional Education, 3(146), 39-44.

Ray, M., \& Jat, K. R. (2010). Effect of electronic media on children. Indian pediatrics, 47(7), 561-568.

Ristkari, T., Kurki, M., Suominen, A., Gilbert, S., Sinokki, A., Kinnunen, M., . . Sourander, A. (2019). WebBased Parent Training Intervention With Telephone Coaching for Disruptive Behavior in 4-YearOld Children in Real-World Practice: Implementation Study. Journal of medical Internet research, 21(4), e11446. 
Rutter, M., Andersen-Wood, L., Beckett, C., Bredenkamp, D., Castle, J., Groothues, C., .. O'Connor, T. G. (1999). Quasi-autistic patterns following severe early global privation. The Journal of Child Psychology and Psychiatry and Allied Disciplines, 40(4), 537-549.

Rutter, M., Kreppner, J., Croft, C., Murin, M., Colvert, E., Beckett, C., . . Sonuga - Barke, E. (2007). Early adolescent outcomes of institutionally deprived and non-deprived adoptees. III. Quasi autism. Journal of Child Psychology and Psychiatry, 48(12), 1200-1207.

Sadeghi, S., Pouretemad, H., Khosrowabadi, R., Fathabadi, J., \& Nikbakht, S. (2019a). Behavioral and electrophysiological evidence for parent training in young children with autism symptoms and excessive screen-time. Asian journal of psychiatry, 45, 7-12. doi:https://doi.org/10.1016/j.ajp.2019.08.003

Sadeghi, S., Pouretemad, H., Khosrowabadi, R., Fathabadi, J., \& Nikbakht, S. (2019b). Effects of parentchild interaction training on children who are excessively exposed to digital devices: A pilot study. The International Journal of Psychiatry in Medicine, 54(6), 408-423. doi:10.1177/0091217419837070

Sadeghi, S., Pouretemad, H., Khosrowabadi, R., Fathabadi, J., \& Nikbakht, S. (2019c). Parent-child interaction effects on autism symptoms and EEG relative power in young children with excessive screen-time. Early Child Development and Care, 1-10. doi:https://doi.org/10.1080/03004430.2019.1649256

Shire, S. Y., Worthman, L. B., Shih, W., \& Kasari, C. (2020). Comparison of Face-to-Face and Remote Support for Interventionists Learning to Deliver JASPER Intervention with Children Who have Autism. Journal of Behavioral Education, 1-22.

Siller, M., Hutman, T., \& Sigman, M. (2013). A parent-mediated intervention to increase responsive parental behaviors and child communication in children with ASD: A randomized clinical trial. Journal of autism and developmental disorders, 43(3), 540-555.

Slobodin, O., Heffler, K. F., \& Davidovitch, M. (2019). Screen Media and Autism Spectrum Disorder: A Systematic Literature Review. Journal of Developmental \& Behavioral Pediatrics, 40(4), 303-311.

Soltanifar, A., Akbarzadeh, F., Moharreri, F., Soltanifar, A., Ebrahimi, A., Mokhber, N., . . Naqvi, S. S. A. (2015). Comparison of parental stress among mothers and fathers of children with autistic spectrum disorder in Iran. Iranian journal of nursing and midwifery research, 20(1), 93.

Speyer, R., Denman, D., Wilkes-Gillan, S., Chen, Y.-W., Bogaardt, H., Kim, J.-H., . . . Cordier, R. (2018). Effects of telehealth by allied health professionals and nurses in rural and remote areas: a systematic review and meta-analysis. Journal of rehabilitation medicine, 50(3), 225-235.

Stiglic, N., \& Viner, R. M. (2019). Effects of screentime on the health and well-being of children and adolescents: a systematic review of reviews. BMJ open, 9(1), e023191.

Sutherland, R., Trembath, D., \& Roberts, J. (2018). Telehealth and autism: A systematic search and review of the literature. International journal of speech-language pathology, 20(3), 324-336.

Taheri, M. (2013). The effect of new communication technologies on social isolation of children in families. Journal of Behavioral sciences in Asia, 1(1), 61-69.

Vismara, L. A., McCormick, C., Young, G. S., Nadhan, A., \& Monlux, K. (2013). Preliminary findings of a telehealth approach to parent training in autism. Journal of Autism and Developmental Disorders, 43(12), 2953-2969.

Waldman, M., Nicholson, S., \& Adilov, N. (2006). Does television cause autism? Retrieved from

Whiteside-Mansell, L., Ayoub, C., McKelvey, L., Faldowski, R. A., Hart, A., \& Shears, J. (2007). Parenting stress of low-income parents of toddlers and preschoolers: Psychometric properties of a short form of the Parenting Stress Index. Parenting: Science and Practice, 7(1), 26-56. 
Yurika, N. U., Hiroyuki, Y., Hiroki, S., Wakaba, E., Mitsugu, U., Chieko, N., \& Shigeo, K. (2018). Attachment Disorder and Early Media Exposure: Neurobehavioral symptoms mimicking autism spectrum disorder. The Journal of Medical Investigation, 65(3.4), 280-282.

Zamfir, M. T. (2018). The consumption of virtual environment more than 4 hours/day, in the children between 0-3 years old, can cause a syndrome similar with the autism spectrum disorder. Journal of Romanian Literary Studies(13), 953-968. 